\title{
Tridiagonal Matrices with Permanent Values Equal to $k$-Jacobsthal Sequence
}

\author{
Porntip Kasempin ${ }^{1}$, Warin Vipismakul ${ }^{2 *}$, Araya Kaewsuy \\ ${ }^{1,2,3}$ Department of Mathematics, Faculty of Science, Burapha University \\ Chonburi, Thailand \\ ${ }^{*}$ Coressponding auther's email: wasinv [AT]buu.ac.th
}

\begin{abstract}
We provide a proof that the permanents of certain tridiagonal matrices are natural numbers in a $k$ Jacobsthal sequence. As a consequence, such matrices are convertible.
\end{abstract}

Keywords - Permanent, $k$-Jacobsthal Sequence, Convertible Matrix

\section{INTRODUCTION}

There are several studies on representing famous sequences of natural numbers as permanent of matrices. We start the article by discussing a few major ones. In [9] and [10], Bozkurt and Yilmaz consider one type of an $n \times n$ upper Hessenberg matrix of odd order

$$
H_{n}=\left[\begin{array}{cccccccc}
1 & 1 & -1 & & & & & \\
1 & 1 & 1 & 1 & & & 0 & \\
& 1 & 1 & 1 & -1 & & & \\
& & \ddots & \ddots & \ddots & \ddots & & \\
& & & 1 & 1 & 1 & 1 & \\
& & & & 1 & 1 & 1 & -1 \\
& 0 & & & & 1 & 1 & 1 \\
& & & & & & 1 & 1
\end{array}\right]
$$

and define one type of lower Hessenberg matrix

$$
\bar{H}_{n}=\left[\begin{array}{ccccccc}
2 & -1 & & & & & \\
0 & 2 & 1 & & & & 0 \\
1 & 0 & 2 & -1 & & & \\
& 1 & 0 & 2 & 1 & & \\
& & \ddots & \ddots & \ddots & \ddots & \\
& 0 & & 1 & 0 & 2 & (-1)^{n-1} \\
& & & & 1 & 0 & 1
\end{array}\right]
$$

For any natural number $n$. The study shows that permanents of these matrices can be realized as Pell numbers, Fibonacci numbers, Lucas numbers and their sums.

Similarly, in [2], Gulec studies an $n \times n$ tridiagonal matrix

$$
H_{n}(s, t)=\left[\begin{array}{ccccc}
2 s & 2 & & & 0 \\
t & 2 s & 1 & & \\
& t & 2 s & \ddots & \\
& & \ddots & \ddots & 1 \\
0 & & & t & 2 s
\end{array}\right]
$$


and proves that

$$
\operatorname{perH}_{n}(s, t)=\operatorname{perH}_{n}^{n-2}(s, t)=q_{n}(s, t)
$$

where $q_{n}(s, t)$ is the $n$th $(s, t)$ - Pell Lucas number.

As a more intricated example, in [8], Minc considers a $n \times n(0,1)$-matrix for which the $i, j$ entry is 1 when $i-1 \leq j \leq i+k-1$ and 0 otherwise for $k \leq n+1$. Namely,

$$
F(n, k)=\left[\begin{array}{ccccccccc}
1 & 1 & \cdots & 1 & 0 & 0 & 0 & \cdots & 0 \\
1 & 1 & \cdots & 1 & 1 & 0 & 0 & \cdots & 0 \\
0 & 1 & \cdots & 1 & 1 & 1 & 0 & \cdots & 0 \\
\cdots & \cdots & \cdots & \cdots & \cdots & \cdots & \cdots & \cdots & \cdots \\
0 & \cdots & \cdots & 0 & 1 & 1 & \cdots & 1 & 0 \\
0 & \cdots & \cdots & 0 & 0 & 1 & \cdots & 1 & 1 \\
\cdots & \cdots & \cdots & \cdots & \cdots & \cdots & \cdots & \cdots & \cdots \\
0 & \cdots & \cdots & 0 & \cdots & 0 & 1 & 1 & 1 \\
0 & \cdots & \cdots & 0 & \cdots & 0 & 0 & 1 & 1
\end{array}\right] .
$$

His main result states that $\operatorname{per} F(n, k)=g_{n+1}^{k}$, where $g_{n}^{k}$ is the $n^{\text {th }}$ generalized order- $k$ Fibonacci number, for $i=k$.

There are related researches on representing famous sequences as the permanent of tridiagonal matrices in $[1,3,4,5,6,7]$. In this article, we explore another type of tridiagonal matrices whose permanent result to the $k$-Jacobsthal sequence. We then show that the matrices are convertible.

\section{MAIN RESULTS}

Definition 1. Let $G_{n}=\left[g_{i j}\right]_{n \times n}$ be a tridiagonal matrix given by $g_{i i}=k, g_{i+1, i}=1, g_{i, i+1}=2$ for $1 \leq i \leq n$ and 0 otherwise. Thus

$$
G_{n}=\left[\begin{array}{ccccc}
k & 2 & & & 0 \\
1 & k & 2 & & \\
& 1 & k & \ddots & \\
& & \ddots & \ddots & 2 \\
0 & & & 1 & k
\end{array}\right] .
$$

This article aims to prove that this given $G_{n}$ has the permanent value in the $k$-Jacobsthal sequence.

Definition 2. For any natural number $n$, let $S_{n}$ be the symmetric group on $\{1,2,3, \ldots, n\}$ and $j=\left(j_{1}, j_{2}, \ldots, j_{n}\right)$ be the element of this group. Then the permanent of an $n$-square matrix $A=\left[a_{i j}\right]_{n \times n}$ is

$$
\text { perA }=\sum_{\text {allj }} a_{1 j_{1}} a_{2 j_{2}} \ldots a_{n j_{n}},
$$

where the summation extends over all permutations $j$ in $S_{n}$.

Definition 3. Let $n \in \square, k>0$.Then, the $k$-Jacobsthal sequence $\left\{\hat{j}_{k, n}\right\}_{n \in \square}$ is defined by

$$
\hat{j}_{k, n}=k \hat{j}_{k, n-1}+2 \hat{j}_{k, n-2} \text {, }
$$

with initial conditions $\hat{j}_{k, 0}=0, \hat{j}_{k, 1}=1$

Namely, it is the sequence

$$
0,1, k, k^{2}+2, k^{3}+4 k, k^{4}+6 k^{2}+4, k^{5}+8 k^{3}+12 k, \ldots .
$$

Since the permanent of a matrix is as complex as the determinant, we will approach the problem by contractible matrices to gradually compute its value. For this reason, we define the following technical definitions below. 
Definition 4. An $m \times n$ matrix $A=\left[a_{i j}\right]$ with row vectors $r_{1}, r_{2}, \ldots, r_{m}$ is contractible on column $k$ if this column contains exactly two nonzero element.

Definition 5. If $A=\left[a_{i j}\right]$ is contractible on column $k$ whose nonzero entries on column $k$ are $a_{i k}, a_{j k}$, define the contraction of $A$ on column $k$ relative to rows $i$ and $j$ to be an $(m-1) \times(n-1)$ matrix $A_{i j: k}$ obtained from $A$ replacing row $i$ with $a_{i k}, a_{j k}$ and deleting row $j$ and column $k$ is called the contraction of $A$ on column $k$ relative to row $i$ and $j$.

\section{Example 1.}

Consider the matrix

$$
A=\left[\begin{array}{cccc}
1 & 2 & 0 & -1 \\
3 & 4 & 2 & 0 \\
1 & -2 & 3 & 5 \\
0 & 7 & -4 & 2
\end{array}\right] .
$$

Since $a_{22} \neq 0 \neq a_{32} \times A$ is contractible on column 2 . Replacing row 2 with $a_{32} r_{2}+a_{22} r_{3}$, we get

$$
\begin{aligned}
& a_{21}=(-2)(3)+(4)(1)=-2 \\
& a_{22}=(-2)(4)+(4)(-2)=-16 \\
& a_{23}=(-2)(2)+(4)(3)=8 \\
& a_{24}=(-2)(0)+(4)(5)=20,
\end{aligned}
$$

which transform the matrix to

$$
\left[\begin{array}{cccc}
1 & 2 & 0 & -1 \\
-2 & -16 & 8 & 20 \\
1 & -2 & 3 & 5 \\
0 & 7 & -4 & 2
\end{array}\right]
$$

After removing row 3 and column 2, we get

$$
\left[\begin{array}{ccc}
1 & 0 & -1 \\
-2 & 8 & 20 \\
0 & -4 & 2
\end{array}\right]
$$

as contraction of $A$ on column 2 relative to row 2 and 3 .

Proposition 1. Let $A$ be a nonnegative integral matrix of order $n>1$. If $B$ is a contraction of $A$ then

$$
\operatorname{per} A=\operatorname{per} B \text {. }
$$

Theorem 1. Let $n$ be a natural number and $\hat{j}_{k, n}$ be a $k$-Jacobsthal sequence. Then

$$
\operatorname{per} G_{n}=\operatorname{per}_{n}^{n-2}=\hat{j}_{k, n+1},
$$

for which matrices $G_{n}^{r}$ obtained by contracting $G_{n} r$ times for any $1 \leq r \leq n-2$.

Proof We will prove by Mathematical induction. It is obvious when $n=1$ that $G_{1}=[k]$. Therefore,

$$
\operatorname{per} G_{1}=k=\hat{j}_{k, 2} \text {. }
$$

For $n=2$, we see that $G_{2}=\left[\begin{array}{ll}k & 2 \\ 1 & k\end{array}\right]$, so $\operatorname{per} G_{2}=k^{2}+2=\hat{j}_{k, 3}$.

Now for $n>2$, we use induction on $l$, the number of contractions performed to show that 


$$
G_{n}^{l}=\left[\begin{array}{ccccc}
j_{k, l+2} & 2 j_{k, l+1} & & & 0 \\
1 & k & 2 & & \\
& 1 & k & \ddots & \\
& & \ddots & \ddots & 2 \\
0 & & & 1 & k
\end{array}\right],
$$

for any $3 \leq l \leq n-4$.

For $l=1$, since $g_{11}, g_{21} \neq 0$, we can contract $G_{n}$ on column 1 .

Firstly, $r_{1}$ is replaced by $g_{21} r_{1}+g_{11} r_{2}$, and

It follows immediately that

$$
\begin{gathered}
g_{11}=1(k)+k(1)=2 k \\
g_{12}=1(2)+k(k)=k^{2}+2 \\
g_{13}=1(0)+k(2)=2 k \\
g_{14}=1(0)+k(0)=0 \\
\vdots \\
g_{1 n}=1(0)+k(0)=0 .
\end{gathered}
$$

$$
H=\left[\begin{array}{ccccc}
2 k & k^{2}+2 & 2 k & & 0 \\
1 & k & 2 & & \\
& 1 & k & \ddots & \\
& & \ddots & \ddots & 2 \\
0 & & & 1 & k
\end{array}\right] .
$$

Let us remove row 2 and column 1 from $H$ to get the $(n-1) \times(n-1)$ contraction matrix $G_{n}^{1}$,

$$
G_{n}^{1}=\left[\begin{array}{ccccc}
k^{2}+2 & 2 k & & & 0 \\
1 & k & 2 & & \\
& 1 & k & \ddots & \\
& & \ddots & \ddots & 2 \\
0 & & & 1 & k
\end{array}\right]=\left[\begin{array}{ccccc}
j_{k, 3} & 2 j_{k, 2} & & & 0 \\
1 & k & 2 & & \\
& 1 & k & \ddots & \\
& & \ddots & \ddots & 2 \\
0 & & & 1 & k
\end{array}\right] .
$$

Now, assume that

$$
G_{n}^{l}=\left[\begin{array}{ccccc}
j_{k, l+2} & 2 j_{k, l+1} & & & 0 \\
1 & k & 2 & & \\
& 1 & k & \ddots & \\
& & \ddots & \ddots & 2 \\
0 & & & 1 & k
\end{array}\right]
$$

then, $G_{n}^{l}$ is contractible on column 1 , so we replace the first row by $g_{21} r_{1}+g_{11} r_{2}$ to see that

$$
\begin{aligned}
a_{11}= & (1) \hat{j}_{k, l+2}+\hat{j}_{k, l+2}(1) \\
a_{12}= & (1)(2) \hat{j}_{k, l+1}+\hat{j}_{k, l+2}(k) \\
a_{13}= & (1)(0)+\hat{j}_{k, l+2}(2) \\
a_{14}= & (1)(0)+\hat{j}_{k, l+2}(0) \\
& \vdots \\
a_{1 n}= & (1)(0)+\hat{j}_{k, l+2}(0) .
\end{aligned}
$$


This results to the matrix

$$
\bar{G}=\left[\begin{array}{ccccc}
2 \hat{j}_{k, l+2} & k \hat{j}_{k, l+2}+2 \hat{j}_{k, l+1} & 2 \hat{j}_{k, l+2} & & 0 \\
1 & k & 2 & & \\
& 1 & k & \ddots & \\
& & \ddots & \ddots & 2 \\
0 & & & 1 & k
\end{array}\right] .
$$

By removing second row and first column of Equation 1, we obtain

which finish our induction.

$$
G_{n}^{l+1}=\left[\begin{array}{ccccc}
\hat{j}_{k,(l+1)+2} & 2 \hat{j}_{k,(l+1)+1} & & & 0 \\
1 & k & 2 & & \\
& 1 & k & \ddots & \\
& & \ddots & \ddots & 2 \\
0 & & & 1 & k
\end{array}\right],
$$

We recall that $n>2$. By choosing $l+1=n-3$, one can achieve that

$$
G_{n}^{n-3}=\left[\begin{array}{ccc}
\hat{j}_{k, n-1} & 2 \hat{j}_{k, n-2} & 0 \\
1 & k & 2 \\
0 & 1 & k
\end{array}\right] .
$$

Lastly, we contract $G_{n}^{n-3}$ on column 1 to compute the matrix

$$
G_{n}^{n-2}=\left[\begin{array}{cc}
k \hat{j}_{k, n-1}+2 \hat{j}_{k, n-2} & 2 \hat{j}_{k, n-1} \\
1 & k
\end{array}\right]=\left[\begin{array}{cc}
\hat{j}_{k, n} & 2 \hat{j}_{k, n-1} \\
1 & k
\end{array}\right] .
$$

Then finding the permanent

$$
\operatorname{perG}_{n}^{n-2}=k \hat{j}_{k, n}+2 \hat{j}_{k, n-1}=\hat{j}_{k, n+1} .
$$

It follows immediately from Proposition 1 that

$$
\operatorname{per} G_{n}=\operatorname{per} G_{n}^{n-2}=\hat{j}_{k, n+1} .
$$

As an application of Theorem 1, we use is to prove an interesting property of $G_{n}$.

Definition 6. An $n \times n$ matrix $A$ is convertible if there is an $(1,-1)$ matrix $K$ such that

$$
\operatorname{per} A=\operatorname{det}(A \circ K) \text {, }
$$

where $A \circ K$ denotes the Hadamard product of $A$ and $K$.

Theorem 2. For any natural number $n, G_{n}$ is convertible.

Proof. Let $S_{n}$ be a $(1,-1)$ matrix of dimension $n \times n$ defined by

$$
S_{n}=\left[\begin{array}{ccccc}
1 & 1 & \cdots & 1 & 1 \\
-1 & 1 & \cdots & 1 & 1 \\
1 & -1 & \cdots & 1 & 1 \\
\vdots & \vdots & \ddots & \vdots & \vdots \\
1 & 1 & \cdots & -1 & 1
\end{array}\right]
$$

Then the Hadamard Product of $G_{n}$ and $S_{n}$ is 


$$
G_{n} \circ S_{n}=\left[\begin{array}{cccccc}
k(1) & 2(1) & 0(1) & 0(1) & \cdots & 0(1) \\
1(-1) & k(1) & 2(1) & 0(1) & \cdots & 0(1) \\
0(1) & 1(-1) & k(1) & 2(1) & \ddots & \vdots \\
0(1) & 0(1) & 1(-1) & k(1) & \ddots & 0(1) \\
\vdots & \vdots & \ddots & \ddots & \ddots & 2(1) \\
0(1) & 0(1) & \cdots & 0(1) & 1(-1) & k(1)
\end{array}\right]=\left[\begin{array}{ccccc}
k & 2 & & & 0 \\
-1 & k & 2 & & \\
& -1 & k & \ddots & \\
& & \ddots & \ddots & 2 \\
0 & & & -1 & k
\end{array}\right] .
$$

We use strong induction on $n$.

Base case: for $n=1, G_{1} \circ S_{1}=[k]$. Hence $\operatorname{det}\left(G_{1} \circ S_{1}\right)=k=j_{k, n}$.

Now assume that $\operatorname{det}\left(G_{r} \circ S_{r}\right)=j_{r, r+1}$ for any $1<r \leq k$.

Now consider

$$
\begin{aligned}
\operatorname{det}\left(G_{k+1} \circ S_{k+1}\right) & =a_{k+1, k+1} \operatorname{det}\left(G_{k} \circ S_{k}\right)-a_{k+1, k} a_{k, k+1} \operatorname{det}\left(G_{k-1} \circ S_{k-1}\right) \\
& =k j_{k, k+1}+2 j_{k, k} \\
& =j_{k, k+2} \\
& =j_{k,(k+1)+1} .
\end{aligned}
$$

Therefore, $\operatorname{det}\left(G_{n} \circ S_{n}\right)=j_{k, n+1}$. By Theorem 1, we obtain

$$
\operatorname{det}\left(G_{n} \circ S_{n}\right)=\operatorname{per} G_{n},
$$

which finishes the proof.

\section{REFERENCES}

[1] Brualdi, R. A., Gibson, P. M., "Convex polyhedra of doubly stochastic matrices I. Applications of permanent function”, Journal of Combinatorial Theory (A), vol.22, pp.194 - 230, 1977.

[2] Gulec, H. H., "Permanents and Determinants of Tridiagonal Matrices with (s,t) Pell Lucus Numbers", International Mathematical Forum, vol.11, no.23, pp.1117 - 1122, 2017.

[3] Jina, J., Trojovsky, P., "On Permanents of some tridiagonal matrices connected with Fibonacci numbers", International Journal of Pure and Applied Mathematics, vol.97, no.1, pp.79 - 87, 2014.

[4] King, B. W., Parker, D. F., "A Fibonacci Matrix and the Permanent Function. The Fibonacci Quarterly”, vol.7, no.5, pp.539- 544, 1969.

[5] Kilic, E., Tasci, D., "On the Permanents of some Tridiagonal Matrices with Applications to the Fibonacci and Lucas numbers”, Rocky Mountain Journal of Mathematics, vol.37, no.6, pp.1953 - 1969, 2007.

[6] Kaygisiz, K., Sahin, A., "Determinant and Permanent of Hessenberg Matrix and Fibonacci Type Numbers", Gen. Math. Notes, vol.9, no.2, pp.32 - 41, 2012.

[7] Lee, G. Y. (2000). K-Lucas numbers and associated bipartite graphs. Linear Algebra and Its Applications, vol.320, pp.51-61, 2000.

[8] Minc, H., "Permanent of $(0,1)$ - circulants", Canad. Math. Bull, vol.7, pp.253 - 263, 1964.

[9] Yilmaz, F., Bozkurt, D, "Hessenberg matrices and the Pell and Perrin numbers", Journal of Number Theory, vol.131, pp.1390 - 1396, 2011.

[10] Yilmaz, F., Bozkurt, D, "On the Fibonacci and Lucas numbers, their sums and permanents of one type of Hessenberg Matrices”, arXiv:1302.0668 [math.NT], 2013. 